\title{
ENHANCEMENT OF STOCHASTIC RESONANCE USING OPTIMIZATION THEORY*
}

\author{
XINGXING WU ${ }^{\dagger}$, ZHONG-PING JIANG ${ }^{\ddagger}$, DANIEL W. REPPERGER ${ }^{\S}$, AND YI GUO
}

\begin{abstract}
The traditional stochastic resonance is realized by adding an optimal amount of noise, while the parameter-tuning stochastic resonance is realized by optimally tuning the system parameters. This paper reveals the possibility to further enhance the stochastic resonance effect by tuning system parameters and adding noise at the same time using optimization theory. The further improvement of the maximal normalized power norm of the bistable double-well dynamic system with white Gaussian noise input can be converted to an optimization problem with constraints on system parameters and noise intensity, which is proven to have one and only one local maximum for the Gaussian-distributed weak input signal. This result is then extended to the arbitrary weak input signal case. For the purpose of practical implementation, a fast-converging optimization algorithm to search the optimal system parameters and noise intensity is also proposed. Finally, computer simulations are performed to verify its validity and demonstrate its potential applications in signal processing.
\end{abstract}

Key words: Optimization, Stochastic Resonance, Signal Processing

1. Introduction. Since put forward by Benzi in 1981, stochastic resonance has been increasingly attracting the interest of researchers. It is a phenomenon of certain nonlinear systems in which the synchronization between the input signal and the noise occurs when an optimal amount of additional noise is inserted into the system. In this case, the extra noise will help, rather than hinder, the performance improvement of the system by maximizing or minimizing the chosen performance measure, such as output signal-to-noise ratio (SNR), or mutual information. The concept of stochastic resonance was first proposed to address the problem of the periodically recurrent ice ages [1]. Basically, the stochastic resonance has four elements: nonlinear system, information-carrying input signal, noise, and performance measure [2]. Many kinds of nonlinear systems have demonstrated the stochastic resonance phenomena, such as static systems [3], dynamic systems [4][19], discrete systems [21], and coupled systems [4][20]. The traditional stochastic resonance requires the information-carrying signal to be weak and periodic. Now, aperiodic and suprathreshold signals can also be the input of certain stochastic resonance systems, in terms of aperiodic stochastic

*This work has been partially supported by the Polytechnic CATT Center sponsored by New York State, NSF grants ECS-009317, OISE-0408925 and DMS-0504462, and an Air Force contract.

†Xingxing Wu is with the Department of Electrical and Computer Engineering, Polytechnic University, Brooklyn, NY 11201, USA. E-mail: xwu03@utopia.poly.edu

$\ddagger$ Zhong-Ping Jiang is with the Department of Electrical and Computer Engineering, Polytechnic University, Brooklyn, NY 11201, USA. E-mail: zjiang@control.poly.edu

$\S$ Daniel W. Repperger is with the Air Force Research Laboratory, AFRL/HECP, Wright-Patterson AFB, OH 45433, USA. E-mail: Daniel.Repperger@wpafb.af.mil

TYi Guo is with the Department of Electrical and Computer Engineering, Stevens Institute of Technology, Hoboken, NJ 07030, USA. E-mail: yguo1@stevens.edu 
resonance (ASR) [5] and suprathreshold stochastic resonance (SSR) [6] respectively. For the noise, it is no longer limited to white Gaussian noise. It can be colored [7], or non-Gaussian noise [8]. The performance measure is adopted here in order to quantify the stochastic resonance phenomenon and describe it more exactly. For the periodic stochastic resonance, the output signal-to-noise ratio [4] is the most commonly used measure. Other measures, such as cross-correlation [9], mutual information [10], are used for describing aperiodic stochastic resonance. Over the years, stochastic resonance has found applications in many different areas, such as in physics, chemistry, biomedical science, and engineering [4][19]. The noise has been used to enhance the tactile sensation [11]. The suprathreshold stochastic resonance has been applied to cochlear implant coding [12]. One of the important applications of stochastic resonance is in signal processing, such as signal detection [13][22], signal transmission [14][15], and signal estimation [16]. For a stochastic resonance system, the chosen performance measure will reach its maximum or minimum when the synchronization between input signal and the noise occurs. In order to convert noise to a positive factor, the stochastic resonance system should be adjusted properly to maximize or minimize the performance measure, such as the signal-to-noise ratio. For traditional stochastic resonance systems, this is realized by supplementing the input with an optimal amount of additional noise [4]. Recently, it was demonstrated that the stochastic resonance effect can also be realized by tuning the system parameters to their optimal values without adding noise. This is called parameter-tuning stochastic resonance [17][23]. It is also shown that tuning system parameters is a better method in some situations than adding noise, especially when the initial noise intensity is already beyond the resonance region [18]. Among this research, either noise is added, or the system parameters are tuned, but not both. This paper will focus on investigating the possibility to further enhance the stochastic resonance effect by tuning system parameters and adding noise at the same time, based on our initial research results in [26]. This enhancement will cause the chosen performance measure to be further increased or decreased and will, in turn, have the potential to further improve the system performance.

The rest of the paper is organized as follows. In Section 2, the normalized power norm of the bistable double-well dynamic system with a Gaussian-distributed weak input signal is proven to reach a higher maximum by tuning system parameters and adding noise at the same time, compared with that by either tuning system parameters or adding noise. This result will be extended to the situation with an arbitrary weak input signal in Section 3. In order to meet the high-speed requirement of some tasks, Section 4 proposes a fast-converging optimization algorithm to search the optimal system parameters and noise intensity. Section 5 will focus on verifying, via computer simulations, the improvement of the maximal normalized power norm by comparing with the traditional stochastic resonance and parameter-tuning stochastic resonance. 
The potential application of this scheme in signal processing is also mentioned in this section. Finally, Section 6 concludes the paper with some brief remarks.

\section{Enhancement of Stochastic Resonance with Gaussian-Distributed} Weak Input Signals. In traditional stochastic resonance systems, the chosen performance measure is taken as a function of the noise intensity, while it is only taken as a function of the system parameters by fixing the noise intensity at its initial level in parameter-tuning stochastic resonance systems. In some stochastic resonance systems, the performance measure is affected by both the system parameters and the noise intensity [24]. This, however, does not necessarily mean that it is possible to enhance the stochastic resonance effect by tuning the system parameters and adding noise at the same time. We have demonstrated this in [26]. Now, we will examine the interesting situation where the stochastic resonance can be further enhanced by tuning the system parameters and adding noise at the same time.

In this paper, we will choose the nonlinear bistable double-well dynamic system as the stochastic resonance system. This is a typical stochastic resonance system which has been extensively used in the work of others [2][4][9][19]. As shown in past literature, the bistable double-well systems have found several applications in signal processing. In [27], it is used to amplify the coherent signals. As a nonlinear filter, it is also used to recover the multi-frequency signals corrupted by noise [17]. Reducing the bit-error rate (BER) of the binary signal transmission is another application of this system [28].

In [9], the aperiodic stochastic resonance was demonstrated in the following nonlinear bistable double-well dynamic system

$$
\frac{d x}{d t}=-\frac{\partial U}{\partial x}+\xi(t)
$$

where $U(x)=-[A-S(t)] x^{2} / 2+x^{4} / 4$ is the symmetric potential function with a fluctuating barrier. $A$ is taken as a positive system parameter in this paper and is used to shift the input signal. $S(t)$ is the input signal with zero-mean average. $\xi(t)$ is white Gaussian noise with zero mean and an autocorrelation of $\langle\xi(t) \xi(s)\rangle=2 D \delta(t-s)$. The angular brackets denote the ensemble average.

The performance measures chosen to describe this aperiodic stochastic resonance are cross-correlation measures (power norm $C_{0}$ and normalized power norm $C_{1}$ ) defined as follows [9]

$$
\begin{gathered}
C_{0}=\max \{\overline{S(t) R(t+\tau)}\}, \\
C_{1}=\frac{C_{0}}{\left[\overline{S^{2}(t)}\right]^{1 / 2}\left\{\overline{[R(t)-\overline{R(t)}]^{2}}\right\}^{1 / 2}},
\end{gathered}
$$


where $S(t)$ is the input signal. $R(t)$ is the system response characterized by mean transition rate of the system. The overbar denotes an average over time. $\tau$ is a time lag.

Usually, there is no explicit expression for the power norm. If the input signal is weak, (i.e., $\overline{S(t)^{2}} \ll A^{2}$ ), and is a Gaussian distribution, the ensemble averaged power norm $\left\langle C_{0}\right\rangle$ and the ensemble averaged normalized power norm $\left\langle C_{1}\right\rangle$ can be approximated by [9]

$$
\left\langle C_{1}\right\rangle \simeq \frac{\Delta_{0}\left[\overline{S^{2}(t)}\right]^{1 / 2}}{\left\{\exp \left[\Delta_{0}^{2} \overline{S^{2}(t)}\right]-1+\sigma(D) Q_{0}^{-2} \exp \left[2 \Theta_{0}-\Delta_{0}^{2} \overline{S^{2}(t)}\right]\right\}^{1 / 2}},
$$

where

$$
\begin{aligned}
\sigma(D) & =K_{1}\langle\overline{R(t)}\rangle, \quad\langle\overline{R(t)}\rangle \simeq Q_{0} \exp \left[-\Theta_{0}+\Delta_{0}^{2} \overline{S^{2}(t) / 2}\right], \\
Q_{0} & =K_{0} A / \sqrt{2} \pi, \quad \Theta_{0}=A^{2} / 4 D, \quad \Delta_{0}=A / 2 D .
\end{aligned}
$$

According to [9], the parameter $K_{0}$ is used to account for normalization factors in the construction of the mean transition rate. The parameter $K_{1}$ is used for the estimation of the noise-induced variance $\sigma(D)$. In this paper, we will set them to the same constant values as in [9], i.e., $K_{0}=1, K_{1}=0.019$.

If the system parameter $A$ is fixed, the normalized power norm $\left\langle C_{1}\right\rangle$ will reach its maximal value when an optimal amount of noise is added into this nonlinear system [9]. We will now investigate whether the stochastic resonance effect of this system can be further enhanced by tuning the system parameters and adding noise at the same time. This is in fact a problem of checking whether the optimization problem of maximizing $\left\langle C_{1}\right\rangle$ with the constraints on both the system parameters and noise intensity has a local maximizer. The computer simulations, however, show that this constrained optimization problem has no local maximizer for some weak input signals. So two additional parameters are introduced into the system and the new system equation becomes

$$
\tau_{a} \dot{x}(t)=[A-S(t)] x(t)-\frac{x^{3}(t)}{X_{b}^{2}}+\xi(t)
$$

where $\tau_{a}$ is a positive system parameter affecting the system response time and $X_{b}$ is a positive system parameter affecting the barrier height of its potential function.

For the system described by (6), its potential function is

$$
U(x)=-[A-S(t)] \frac{x^{2}}{2}+\frac{x^{4}}{4 X_{b}^{2}} .
$$

If $\tau_{a}=1$, the ensemble-averaged escape rate is expressed as [9]

$$
\langle R(t)\rangle \simeq \frac{1}{2 \pi} \sqrt{U^{\prime \prime}\left(x_{\min }\right)\left|U^{\prime \prime}\left(x_{\max }\right)\right|} \exp \left[\frac{U\left(x_{\min }\right)-U\left(x_{\max }\right)}{D}\right],
$$


where $U$ is the potential function, $x_{\min }$ is one of the local minimizers and $x_{\max }$ is the local maximizer of the potential function.

Using the method of [9], similar to (5), we derive the approximation expression of $\left\langle C_{1}\right\rangle$ for system (6)

$$
\left\langle C_{1}\right\rangle \simeq \frac{\Delta s}{\left\{\exp \left[\Delta^{2} s^{2}\right]-1+K_{1} Q^{-1} \exp \left[c \tau_{a} \Delta Q-s^{2} \Delta^{2} / 2\right]\right\}^{1 / 2}},
$$

where

$$
Q=\frac{K_{0} A}{\sqrt{2} \tau_{a} \pi}, \Theta=\frac{\tau_{a} X_{b}^{2} A^{2}}{4 D}=c \tau_{a} \Delta Q, \Delta=\frac{\tau_{a} X_{b}^{2} A}{2 D}, s=\left[\overline{S^{2}(t)}\right]^{1 / 2}, c=\sqrt{2} \pi / 2 K_{0} .
$$

We will choose the normalized power norm $\left\langle C_{1}\right\rangle$ as the objective function to be maximized, because it emphasizes the similarity between the input and the system output, and it can still predict its real shape even when the noise intensity is outside its validity range [9]. The enhancement of stochastic resonance can then be converted to finding the local maximizer of the following optimization problem

$$
\begin{aligned}
& \qquad \max \left\langle C_{1}\right\rangle, \\
& \text { subject to: } \overline{S(t)^{2}} \ll A^{2}, A>0, \quad D_{0} \leq D \leq D_{1} .
\end{aligned}
$$

The constraint $\overline{S(t)^{2}} \ll A^{2}$ comes from the requirement of weak input signals. The system parameter $A$ is positive. We assume that the noise cannot be removed from the system, so the noise intensity $D$ cannot be less than its initial value $D_{0}$. Of course, it cannot be arbitrarily large either.

Both $A$ and $D$ will be taken as the optimization parameters for this optimization problem (10), while $\tau_{a}$ and $X_{b}$ are taken as the supporting parameters to ensure that this constrained optimization problem has local maximizer as shown later. To simplify the derivation and calculation, the direct optimization parameters of (10) are $\Delta$ and $Q$, from which the values of $A$ and $D$ can be calculated.

In order to prove that the constrained optimization problem (10) has a local maximizer, we will first consider the following unconstrained optimization problem

$$
\max \left\langle C_{1}\right\rangle \text {. }
$$

Proposition 1. There is one and only one solution $\left(Q^{*}, \Delta^{*}\right)$ to the first-order necessary condition for the local maximizer of unconstrained optimization problem (11).

Proof. According to the first-order necessary condition for the local maximizer of 

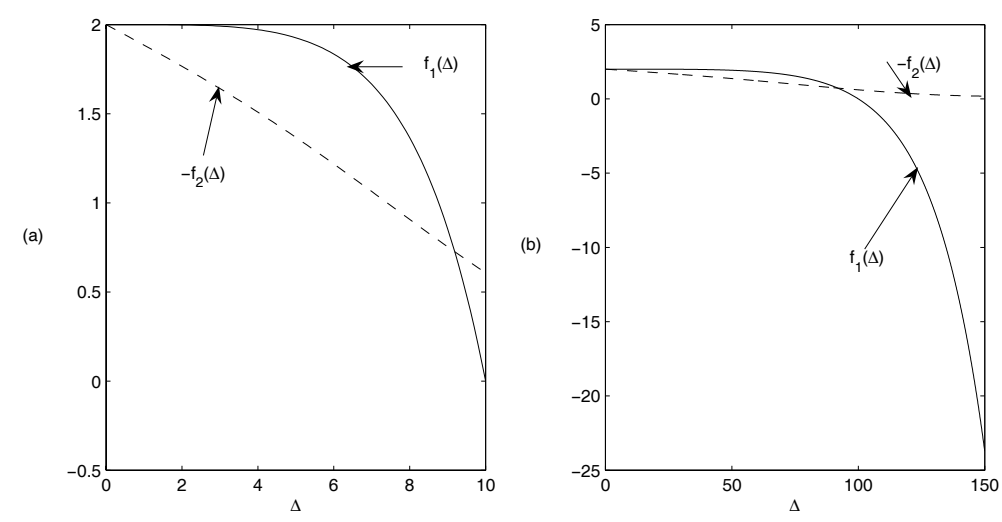

FIG. 1. $f_{1}(\Delta)$ and $-f_{2}(\Delta)$ (a) $\tau_{a}=1, s=0.1$ (b) $\tau_{a}=0.1, s=0.01$.

(11), we have

$$
\frac{\partial\left\langle C_{1}\right\rangle}{\partial \Delta}=0 \quad \text { and } \quad \frac{\partial\left\langle C_{1}\right\rangle}{\partial Q}=0
$$

From (12), we can derive $Q^{-1}=c \tau_{a} \Delta$, and also

$$
\left(2-2 s^{2} \Delta^{2}\right) \exp \left[s^{2} \Delta^{2}\right]-2+c \tau_{a} K_{1}\left(\Delta+s^{2} \Delta^{3}\right) \exp \left[1-s^{2} \Delta^{2} / 2\right]=0 .
$$

Let

$$
f(\Delta)=\left(2-2 s^{2} \Delta^{2}\right) \exp \left[s^{2} \Delta^{2}\right]-2+c \tau_{a} K_{1}\left(\Delta+s^{2} \Delta^{3}\right) \exp \left[1-s^{2} \Delta^{2} / 2\right] .
$$

We have

$$
f^{\prime}(\Delta)=-4 s^{4} \Delta^{3} \exp \left[s^{2} \Delta^{2}\right]+c \tau_{a} K_{1}\left(1+2 s^{2} \Delta^{2}-s^{4} \Delta^{4}\right) \exp \left[1-s^{2} \Delta^{2} / 2\right] .
$$

From (14) and (15), we know that $f(0)=0$ and $f(+\infty)=-\infty . f^{\prime}(\Delta)$ will be positive, if $\Delta \rightarrow 0^{+}$. Based on these, we can conclude the first-order necessary condition has at least one positive solution $\left(\Delta^{*}, Q^{*}\right)$.

Let

$$
\begin{aligned}
& f_{1}(\Delta)=\left(2-2 s^{2} \Delta^{2}\right) \exp \left[s^{2} \Delta^{2}\right], \\
& f_{2}(\Delta)=c \tau_{a} K_{1} \Delta\left(1+s^{2} \Delta^{2}\right) \exp \left[1-s^{2} \Delta^{2} / 2\right]-2 .
\end{aligned}
$$

$f_{1}(\Delta)$ will decrease monotonically to $-\infty$ as $\Delta \rightarrow+\infty$, starting from $f_{1}(0)=2 . f_{2}(\Delta)$ will first increase from -2 , and then decrease to -2 . From the special characteristics of $f_{1}(\Delta)$ and $f_{2}(\Delta)$, it follows readily that the first-order necessary condition can only 

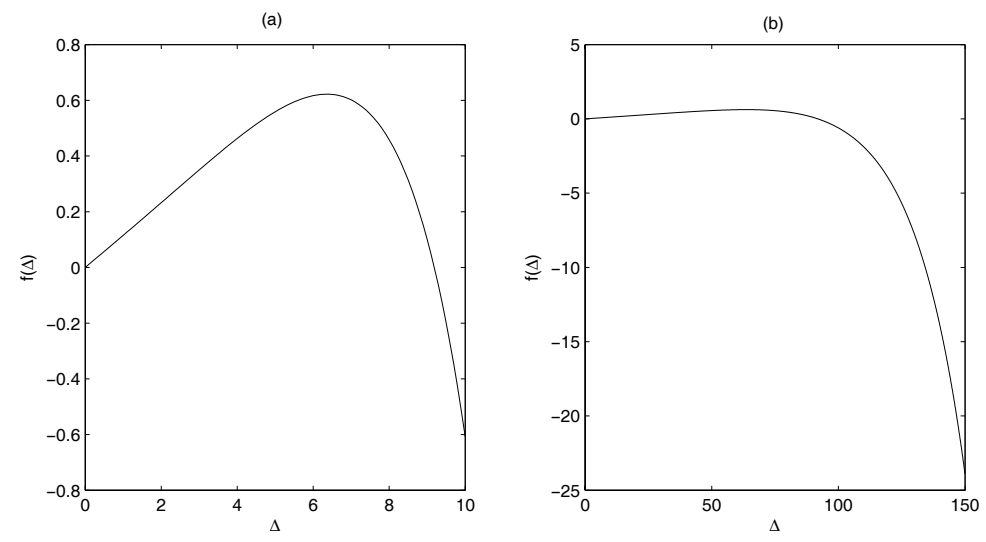

FIG. 2. $f(\Delta)$ (a) $\tau_{a}=1, s=0.1(b) \tau_{a}=0.1, s=0.01$

have one positive solution.

It is worth noting that changing the values of $\tau_{a}$ and $s$ which are positive can only affect the values of $Q^{*}$ and $\Delta^{*}$. It will not affect the property that the necessary condition has one and only one solution. This is shown in Figures 1 and 2.

Proposition 2. The system parameter $\tau_{a}$ can be used to continuously adjust the value of $\Delta^{*}$ satisfying the first-order necessary condition for the local maximizer of (11).

Proof. $f_{2}(\Delta)$, but not $f_{1}(\Delta)$, is affected by the system parameter $\tau_{a}$. From the special characteristics of these two functions, we can find out that the increase of $\tau_{a}$ value will also increase the value of $\Delta^{*}$ satisfying (13). If $\tau_{a}$ is getting close to zero, $\Delta^{*}$ will also approach zero. This means the value of $\Delta^{*}$ can be adjusted continuously by changing $\tau_{a}$. This completes the proof of this proposition.

Proposition 3. The unconstrained optimization problem (11) has one and only one local maximizer when the input is weak $(s \ll 1)$ and the values of system parameters $\tau_{a}$ and $X_{b}$ are chosen properly.

Proof. We need to prove that the only solution $\left(\Delta^{*}, Q^{*}\right)$ of the first-order necessary condition will also satisfy the second-order sufficient condition for a local maximizer, that is, the Hessian matrix is negative definite at the point $\left(\Delta^{*}, Q^{*}\right)$. 
At the point $\left(\Delta^{*}, Q^{*}\right)$, we can get

(18) $\frac{\partial^{2}\left\langle C_{1}\right\rangle}{\partial \Delta^{2}}=\frac{-2 s^{3} \Delta^{* 2}\left\{s \Delta^{*} \exp \left[s^{2} \Delta^{* 2}\right]+c \tau_{a} K_{1}\left(-\frac{1}{2}+\frac{s^{2} \Delta^{* 2}}{4}\right) \exp \left[1-\frac{s^{2} \Delta^{* 2}}{2}\right]\right\}}{\left\{\exp \left[s^{2} \Delta^{* 2}\right]-1+K_{1} Q^{*-1} \exp \left[1-\frac{s^{2} \Delta^{* 2}}{2}\right]\right\}^{3 / 2}}$,

(19) $\frac{\partial^{2}\left\langle C_{1}\right\rangle}{\partial Q^{2}}=\frac{-c^{3} \tau_{a}^{3} s K_{1} \Delta^{* 4} \exp \left[1-\frac{s^{2} \Delta^{* 2}}{2}\right]}{2\left\{\exp \left[s^{2} \Delta^{* 2}\right]-1+K_{1} Q^{*-1} \exp \left[1-\frac{s^{2} \Delta^{* 2}}{2}\right]\right\}^{3 / 2}}$,

(20) $\frac{\partial^{2}\left\langle C_{1}\right\rangle}{\partial \Delta \partial Q}=\frac{\partial^{2}\left\langle C_{1}\right\rangle}{\partial Q \partial \Delta}=\frac{-c^{2} \tau_{a}^{2} s K_{1} \Delta^{* 2} \exp \left[1-\frac{s^{2} \Delta^{* 2}}{2}\right]}{2\left\{\exp \left[s^{2} \Delta^{* 2}\right]-1+K_{1} Q^{*-1} \exp \left[1-\frac{s^{2} \Delta^{* 2}}{2}\right]\right\}^{3 / 2}}$.

The numerator of $\frac{\partial^{2}\left\langle C_{1}\right\rangle}{\partial \Delta^{2}}$ can be proven to be negative for the weak input signal $(s \ll 1)$, i.e.

$$
\begin{aligned}
& -2 s^{2} \Delta^{* 2} \exp \left[s^{2} \Delta^{* 2}\right]-2 c \tau_{a} s K_{1} \Delta^{*}\left(-\frac{1}{2}+\frac{s^{2} \Delta^{* 2}}{4}\right) \exp \left[1-\frac{s^{2} \Delta^{* 2}}{2}\right] \\
& =-2 \exp \left[s^{2} \Delta^{* 2}\right]+2-c \tau_{a} K_{1} \Delta^{*}\left[1-s+s^{2} \Delta^{* 2}\left(1+\frac{s}{2}\right)\right] \exp \left[1-\frac{s^{2} \Delta^{* 2}}{2}\right] \\
& \simeq-2 s^{2} \Delta^{* 2} \exp \left[s^{2} \Delta^{* 2}\right]<0 .
\end{aligned}
$$

From (18), (19), and (20), it follows that $\frac{\partial^{2}\left\langle C_{1}\right\rangle}{\partial \Delta^{2}}, \frac{\partial^{2}\left\langle C_{1}\right\rangle}{\partial Q^{2}}$, and $\frac{\partial^{2}\left\langle C_{1}\right\rangle}{\partial \Delta \partial Q}$ are all negative at $\Delta=\Delta^{*}$, and $Q=Q^{*}$.

The Hessian matrix of the optimization problem (11) is defined as

$$
\left(\begin{array}{ll}
\frac{\partial^{2}\left\langle C_{1}\right\rangle}{\partial \Delta^{2}} & \frac{\partial^{2}\left\langle C_{1}\right\rangle}{\partial \Delta \partial Q} \\
\frac{\partial^{2}\left\langle C_{1}\right\rangle}{\partial Q \partial \Delta} & \frac{\partial^{2}\left\langle C_{1}\right\rangle}{\partial Q^{2}}
\end{array}\right)
$$

At the point $\left(\Delta^{*}, Q^{*}\right)$, the determinant value of this Hessian matrix is

$$
\frac{c^{3} \tau_{a}^{3} K_{1} s^{2} \Delta^{4}\left\{2 \exp \left[s^{2} \Delta^{* 2}\right]-2+c \tau_{a} K_{1}\left(\Delta^{*}-\frac{1}{s \Delta^{*}}+s^{2} \Delta^{* 3}\right) \exp \left[1-\frac{s^{2} \Delta^{* 2}}{2}\right]\right\}}{4\left\{\exp \left[s^{2} \Delta^{* 2}\right]-1+K_{1} Q^{*-1} \exp \left[1-\frac{s^{2} \Delta^{* 2}}{2}\right]\right\}^{3}}
$$

According to Proposition 2, the requirement $s \Delta^{* 2} \gg 1$ can be satisfied by properly adjusting the system parameter $\tau_{a}$ value. In this situation, the determinant value will be positive, because of

$$
\begin{aligned}
& 2 \exp \left[s^{2} \Delta^{* 2}\right]-2+c \tau_{a} K_{1}\left(\Delta^{*}-\frac{1}{s \Delta^{*}}+s^{2} \Delta^{* 3}\right) \exp \left[1-\frac{s^{2} \Delta^{* 2}}{2}\right] \\
& \simeq 2 \exp \left[s^{2} \Delta^{* 2}\right]-2+c \tau_{a} K_{1}\left(\Delta^{*}+s^{2} \Delta^{* 3}\right) \exp \left[1-\frac{s^{2} \Delta^{* 2}}{2}\right] \\
& =2 s^{2} \Delta^{* 2} \exp \left[s^{2} \Delta^{* 2}\right]>0
\end{aligned}
$$

Obviously, its denominator is positive. From the standard test on negativedefiniteness of a symmetric matrix, it follows that the Hessian matrix is negative definite. This completes the proof of Proposition 3. 
Proposition 4. The constrained optimization problem (10) with weak input signal $(s \ll 1)$ has one and only one local maximizer, if the system parameters $\tau_{a}$ and $X_{b}$ are chosen properly.

Proof. From (9), we can get $A^{*}=2 / \Delta^{*}$. The constraint $\left(A^{*}\right)^{2} \gg s^{2}$ will be satisfied if $s^{2}\left(\Delta^{*}\right)^{2} \ll 4$. Combined with the requirements $s\left(\Delta^{*}\right)^{2} \gg 1$ and $s \ll 1$ used for the derivation of the above propositions, all the constraints on the system parameter will be met, if $\tau_{a}$ value is chosen properly such that

$$
s \ll s^{2}\left(\Delta^{*}\right)^{2} \ll 4
$$

Also, $D^{*}$ will be greater than $D_{0}$ and less than $D_{1}$ for the properly chosen parameter $X_{b}$, because of $D^{*}=\tau_{a} X_{b}^{2} / \Delta^{* 2}$.

From these, we prove that the one and only one local maximizer of the unconstrained optimization problem (11) will also be the one and only one local maximizer of the constrained optimization problem (10) for the weak input signal $(s \ll 1)$, when the values of $\tau_{a}$ and $X_{b}$ are chosen properly.

Obviously, the only local maximizer of (10) is also its global maximizer. This completes the proof of this proposition.

According to the above propositions, the normalized power norm $\left\langle C_{1}\right\rangle$ of the bistable double-well system with Gaussian-distributed weak input signals can be maximized by tuning system parameter $A$ and adding noise at the same time and will reach a higher maximal value compared with that by only tuning the system parameter or by only adding noise.

\section{Enhancement of Stochastic Resonance with Arbitrary Weak Input}

Signals. The above propositions are derived under the assumption of only considering Gaussian-distributed weak input signals. Now, we will prove that all these propositions can be extended to the case with arbitrary weak input signals.

Under the condition of $\Delta^{2} \overline{S(t)^{2}} \ll 1$, the normalized power norm $\left\langle C_{1}\right\rangle$ can be approximated by

$$
\left\langle C_{1}\right\rangle \simeq \frac{\Delta s}{\left\{\Delta^{2} s^{2}+\frac{\Delta^{4} s^{4}}{2}+\frac{K_{1}}{Q}\left(1-\frac{\Delta^{2} s^{2}}{2}+\frac{\Delta^{4} s^{4}}{8}\right) \exp \left[c \tau_{a} Q \Delta\right]\right\}^{1 / 2}} .
$$

The related constrained optimization problem is then changed to

$$
\begin{gathered}
\max \left\langle C_{1}\right\rangle, \\
\text { subject to: } A>0, s^{2} \ll A^{2}, \Delta^{2} s^{2} \ll 1, D_{0} \leq D \leq D_{1} .
\end{gathered}
$$

To prove Proposition 1, the following can be derived from the first-order necessary condition for a local maximizer of the optimization problem (11) with the new $\left\langle C_{1}\right\rangle$ 
expression (23):

$$
\begin{gathered}
c \tau_{a} \Delta Q=1, \\
-s^{4} \Delta^{3}+c \tau_{a} e K_{1}\left(1+s^{2} \Delta^{2} / 2-3 s^{4} \Delta^{4} / 8\right)=0 .
\end{gathered}
$$

From this, Proposition 1 and Proposition 2 can be proven in the similar way as before.

In order to prove Proposition 3, the following can be determined if the constraint $s^{2} \Delta^{* 2} \ll 1$ is satisfied by adjusting $\tau_{a}$ properly

$$
-c \tau_{a} e K_{1} s^{2} \Delta^{* 2}+s^{2} \Delta^{*}\left(-4+2 s^{2} \Delta^{* 2}\right)-c \tau_{a} e K_{1} s^{2} \Delta^{* 2}\left(2-3 s^{2} \Delta^{* 2} / 4\right)<0
$$

and

$$
-s^{4} \Delta^{4} / 8+\left(-1+s^{2} \Delta^{* 2} / 2\right)<0
$$

From these, $\frac{\partial^{2}\left\langle C_{1}\right\rangle}{\partial \Delta^{2}}, \frac{\partial^{2}\left\langle C_{1}\right\rangle}{\partial Q^{2}}$, and $\frac{\partial^{2}\left\langle C_{1}\right\rangle}{\partial \Delta \partial Q}$ are all proven to be negative at $\Delta=\Delta^{*}$, and $Q=Q^{*}$.

For (23), the numerator of the Hessian matrix determinant value can be simplified, at $\Delta=\Delta^{*}$ and $Q=Q^{*}$, as

$$
\begin{aligned}
& s^{4} \Delta^{* 3}\left(2-2 s^{2} \Delta^{* 2}\right)+c \tau_{a} e K_{1} s^{4} \Delta^{* 4}\left(\frac{14}{8}-\frac{9 s^{2} \Delta^{* 2}}{8}\right) \\
(29)+ & +\left(s^{4} \Delta^{* 3}+\frac{3 c \tau_{a} e K_{1} s^{4} \Delta^{* 4}}{8}-\frac{c \tau_{a} e K_{1} s^{2} \Delta^{* 2}}{2}\right)+\frac{s^{8} \Delta^{* 7}}{2}+\frac{15 c \tau_{a} e K_{1} s^{8} \Delta^{* 8}}{64} .
\end{aligned}
$$

If $s^{2} \Delta^{* 2} \ll 1$, this numerator value will be positive. Similarly, Proposition 3 is shown to hold for this optimization problem (24).

The constraint $s^{2} \ll A^{* 2}$ will be satisfied if $s^{2} \Delta^{* 2} \ll 1$ is met by properly adjusting $\tau_{a}$, because of $A^{*}=2 / \Delta^{*}$. Proposition 4 is hereby proved for the optimization problem (24).

4. Optimization Algorithms. The constrained optimization problem (24) has no closed-form solution. The optimization algorithm should be developed to search the optimal system parameter and the optimal noise intensity. In order to meet the high-speed requirement of some tasks, a fast-converging optimization algorithm is proposed for (24) with an arbitrary weak input signal in this paper.

Proposition 5. The Newton's method for solving nonlinear equations can be used to search the optimal parameters of the constrained optimization problem (24) with a local Q-quadratic convergence, if the initial value, $\tau_{a}$ and $X_{b}$ values are properly chosen. 
Proof. According to Proposition 4, the constrained optimization problem (24) has one and only one local maximizer $\left(\Delta^{*}, Q^{*}\right)$ which satisfies the equations (25) and (26), if $\tau_{a}$ and $X_{b}$ values are chosen properly. In this case, the optimal parameters $\left(A^{*}, D^{*}\right)$ can be obtained by solving the nonlinear equation (26).

Let

$$
f_{3}(\Delta)=-s^{4} \Delta^{3}+c \tau_{a} e K_{1}\left(1+s^{2} \Delta^{2} / 2-3 s^{4} \Delta^{4} / 8\right) .
$$

$\nabla f_{3}\left(\Delta^{*}\right)$ will be non-singular in this case. According to the standard arguments from [25], the Newton's method can be used to solve (26) with a local Q-quadratic convergence, if the initial value $\Delta^{o}$ is sufficiently close to $\Delta^{*}$.

Based on Proposition 5, our proposed optimization algorithm will first estimate the initial $\Delta^{o}$ value which will be sufficiently close to its optimal value $\Delta^{*}$, then the standard Newton algorithm will be called to search the optimal value. The algorithm is divided into two cases. In the first case, only $A$ and $D$ are adjustable, while the system parameters $\tau_{a}$ and $X_{b}$ are given beforehand. Their values will ensure the existence of the local maximizer for the constrained optimization problem (24). In the second case, the system parameters $\tau_{a}$ and $X_{b}$ should also be optimized.

\section{Case 1:}

In order to estimate the initial values for different input signals, a table is constructed which describes the relationship between the input signal average amplitude value $s=\left[\overline{S^{2}(t)}\right]^{1 / 2}$ and the optimal value $\Delta^{*}$ related to this input. In order to construct the table, we can first select a series of signal average amplitude values. Usually, these values divide the signal average amplitude range evenly. For each signal average amplitude $s$ value, the related optimal value $\Delta^{*}$ satisfying (26) can be obtained off-line using the standard Newton algorithm. Then, the pair $\left(s, \Delta^{*}\right)$ can be inserted into the table. If the algorithm is implemented in software, the table can be represented by a one-dimensional array. For any given input, the related initial value $\Delta^{o}$ can then be estimated based on the information provided by this table, such as using interpolation. It will be sufficiently close to its optimal value to ensure the required convergence speed, if the table is constructed properly. Algorithm 1 is the optimization algorithm for the first case.

\section{Algorithm 1:}

Step 1:

Calculate the average amplitude $s=\left[\overline{S^{2}(t)}\right]^{1 / 2}$ for the given input signal;

Step 2:

Estimate initial $\Delta^{o}$, using the constructed table;

Step 3:

Solve (26) using standard Newton algorithm [25]; 
Step 4:

Calculate $A^{*}$ and $D^{*}$, then stop.

\section{Case 2:}

In this case, system parameters $\tau_{a}$ and $X_{b}$ are unknown and should be optimized for the constrained optimization problem (24). Here, we also assume $\tau_{a} X_{b}^{2}$ is a properly chosen constant which ensures the existence of the local maximizer of (24). The equation (23) reveals that the smaller the $\tau_{a}$ value is, the larger the $\left\langle C_{1}\right\rangle$ will be if other parameters are fixed. This means that there will be no local maximizer for parameter $\tau_{a}$. Also, a smaller $\tau_{a}$ value will generate a smaller $\Delta^{*}$ satisfying (26) and make it easier to satisfy the requirement of $\Delta^{* 2} s^{2} \ll 1$. Too small $\tau_{a}$ value, however, will make it difficult to satisfy the constraints on noise intensity, because of $D^{*}=\tau_{a} X_{b}^{2} / \Delta^{* 2}$. For a given input signal, there will be a range for $\tau_{a}$ value in which the constrained optimization problem (24) will have a local maximizer. The optimal $\tau_{a}$ value will take the extremum. In the following algorithm, we will take the smallest $\tau_{a}$ value as its optimal value, which will ensure the existence of the local maximizer.

Similarly, tables will be constructed to increase this algorithm's convergence speed. Case 2 will have two tables. The first one describes the relationship of $\Delta^{*}$ with input signal average amplitude $s$ and system parameter $\tau_{a}$. The second one describes the relationship between input signal average amplitude $s$ and $\tau_{a}^{*}$ which is the optimal value under this definition related to this input signal. For the first table, we will first select a series of $\left(s, \tau_{a}\right)$ values. For each pair $\left(s, \tau_{a}\right)$, the related $\Delta^{*}$ satisfying $(26)$ can be calculated off-line using standard Newton algorithm. Then the pair $\left(s, \tau_{a}, \Delta^{*}\right)$ is inserted into table one. For the second table, a series of input average amplitude values should also be chosen first. The related optimal value $\tau_{a}^{*}$ can be obtained offline. The pair $\left(s, \tau_{a}^{*}\right)$ is then inserted into table two. If the algorithm is implemented in software, table one can be represented by a two-dimensional array, and table two is represented by a one-dimensional array.

For a given input signal, the optimal $\tau_{a}^{*}$ value can be estimated based on the information provided by table two. It will then be used to estimate $\Delta^{o}$ from table one which will be used as the initial value to solve (26) by Newton algorithm. Both of the estimations can be performed using related interpolation algorithms. The optimal values of $\Delta^{*}, A^{*}$ and $D^{*}$ will then be evaluated to decide how the $\tau_{a}$ value should be changed for next loop. If $\Delta^{* 2} s^{2} \ll 1$ or $D^{*} \geq D_{0}$ is not satisfied, $\tau_{a}$ value will then be decreased. If $D^{*} \leq D_{1}$ is not met, its value will be increased instead. The detailed algorithm is shown in Algorithm 2.

\section{Algorithm 2:}

Step 1: 
Calculate $s=\left[\overline{S^{2}(t)}\right]^{1 / 2}$, set $x_{0}=0, y_{0}=+\infty ;$

Step 2:

Estimate $\tau_{a}^{*}$, take it as the initial value $\tau_{a 0}$;

Step 3:

Estimate the initial value $\Delta^{0}$;

Step 4:

Solve (26) using standard Newton algorithm [25];

Step 5:

If $\tau_{a k}$ is too large:

$y_{k+1}=\tau_{a k}, x_{k+1}=x_{k}, \tau_{a(k+1)}=\frac{\left(x_{k+1}+y_{k+1}\right)}{2}$

else:

$y_{k+1}=y_{k}, x_{k+1}=\tau_{a k}$

if $y_{k+1}=+\infty$ :

$\tau_{a(k+1)}=2 \tau_{a k}$

else:

$\tau_{a(k+1)}=\left(x_{k+1}+y_{k+1}\right) / 2$

Step 6:

If $\left|\tau_{a(k+1)}-\tau_{a k}\right|<\varepsilon:$

If all constraints are satisfied

Calculate $A^{*}$, and $D^{*}$, then stop.

else

No optimal solution

else:

Go back to Step 3

In the above algorithm, the condition "too large" means $D<D_{1}$. In addition, the "no optimal solution" problem can be solved by adjusting $\tau_{a} X_{b}^{2}$ value properly.

The above algorithms are used to search the optimal values of system parameter $A$ and noise intensity $D$ at the same time. In this algorithm, the nonlinear equation (26) should be solved to obtain the optimal value $\Delta^{*}$. It will have no overhead, compared with the algorithms which are used to search either the optimal value of system parameter $A$ or the optimal value of the noise intensity $D$. If only the noise intensity is adjustable and the value of system parameter $A$ is fixed, the optimization algorithm for (24) will only search the optimal value $D^{*}$ to maximize $\left\langle C_{1}\right\rangle$. From (9), we can find that $Q$ will be constant and $\left\langle C_{1}\right\rangle$ will be the function of $\Delta$. From the necessary condition for a local maximizer $\frac{d\left\langle C_{1}\right\rangle}{d \Delta}=0$, we can get a nonlinear equation similar to (26). The optimal value $\Delta^{*}$ can be obtained by solving this equation. From $\Delta^{*}$, we can get the optimal noise intensity $D^{*}$. Similarly, if only the system parameter $A$ is adjustable and the noise intensity $D$ is fixed, the optimization algorithm for (24) 


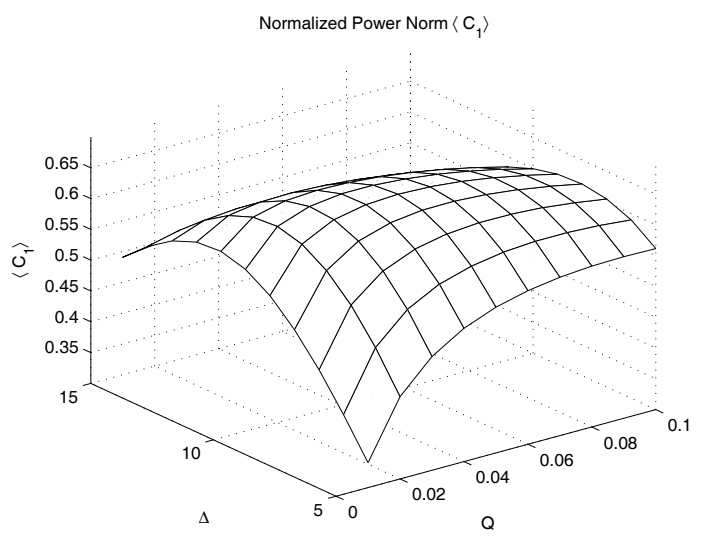

FIG. 3. Normalized power norm $\left\langle C_{1}\right\rangle$.

will only search the optimal value $A^{*}$ to maximize the $\left\langle C_{1}\right\rangle$. Also from (9), we can notice that $\left\langle C_{1}\right\rangle$ will be the function of $Q$. From the necessary condition for a local maximizer $\frac{d\left\langle C_{1}\right\rangle}{d Q}=0$, we can also get a nonlinear equation similar to (26) from which the optimal value $Q^{*}$ can be calculated. The optimal value $A^{*}$ will in turn be obtained. From above analysis, we can conclude that the optimization algorithm for searching both $A^{*}$ and $D^{*}$ will have the same complexity as that for searching either $A^{*}$ or $D^{*}$. The only difference is the nonlinear equation which needs to be solved.

5. Simulations. Computer simulations were performed to verify the above propositions and demonstrate the improvement of the maximal normalized power norm $\left\langle C_{1}\right\rangle$, compared with that by only tuning the system parameter and that by only adding noise. Figure 3 shows that the constrained optimization problem (24) has the local maximizer. Figure 4 compares the maximal $\left\langle C_{1}\right\rangle$ reached by three different methods: (1) only adjusting system parameter $A$ while letting $D=0.1$ (2) only adjusting noise intensity $D$ while letting $A=1$ (3) tuning system parameter $A$ and adjusting the noise intensity $D$ at the same time. From this figure, we can see the enhancement of stochastic resonance effect by this scheme.

Also, computer simulations are performed to reveal its potential application in weak signal recovery. The enhancement of the stochastic resonance effect mentioned above means the similarity between the input signal and the output of the bistable double-well dynamic system with white Gaussian noise input is enhanced. The system output will carry more information about the weak input signal, if it is used for the weak signal recovery. This will make it easier to recover the weak input signal from the noisy system output. Figure 5 is the simulation model. In this model, $A$, $a$, and $b$ are the system parameters and $a=1 / \tau_{a}, b=1 /\left(\tau_{a} X_{b}^{2}\right)$. The noise intensity $D$ will affect the output of the White Noise block. The Constant block with value "shift" is used to shift the average value of the input pulse to zero. The User-Defined Functions 


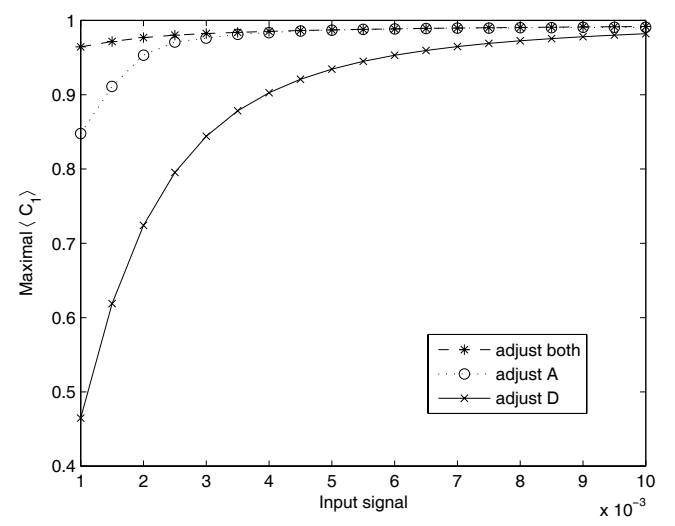

FIG. 4. Comparison of maximal $\left\langle C_{1}\right\rangle$.

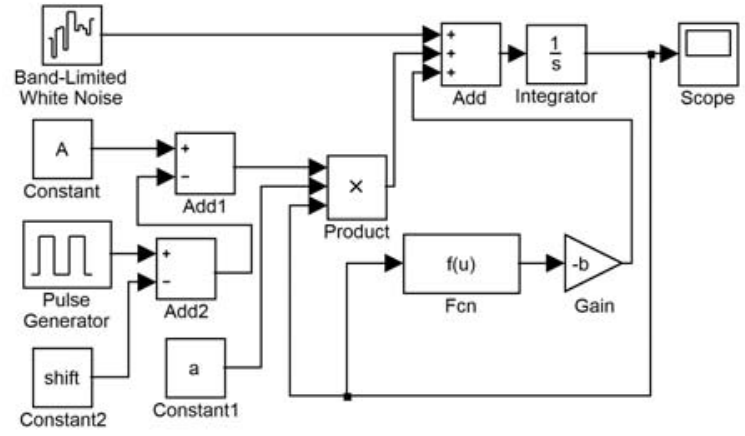

FiG. 5. Simulation model.

block $f(u)$ is used to generate $x^{3}$. Figure 6 is the original weak input signal and the white noise. The system outputs under different system parameter values and different noise intensity are shown in Figure 7. From this simulation, it is obvious that the similarity between input and output, or the input signal information carried by the system output, is greatly affected by the choices of the system parameters and noise intensity. The weak input signal can be better recovered from the noisy system output when the system parameter values and noise intensity are chosen properly.

6. Conclusion. This paper explicitly reveals that it is possible to further enhance the stochastic resonance effect of the bistable double-well dynamic system with white Gaussian noise input by tuning system parameters and adding noise at the same time. The fast-converging optimization algorithms introduced enable this scheme to be applied into applications with high-speed requirements. The enhancement of stochastic resonance effect means the enhancement of the similarity between the in- 


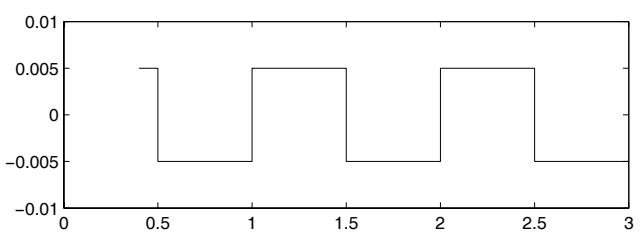

(a)

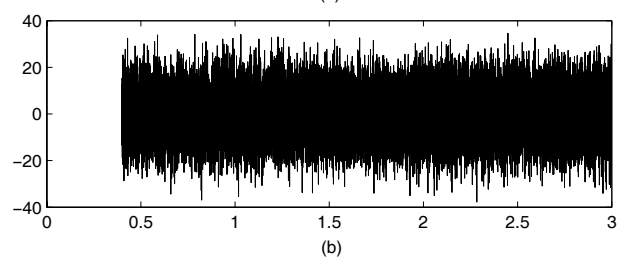

FIG. 6. Input signal and noise (a) input signal (b) white noise.
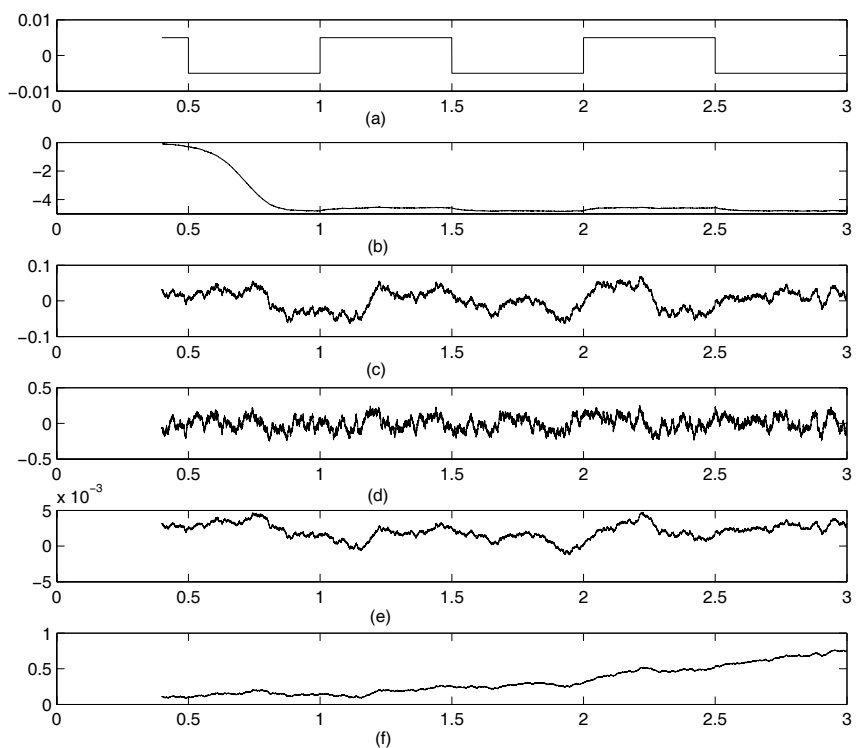

FIG. 7. Input (a) original input signal, and system output for different parameter values (b) $A=0.11, a=100, b=0.5, D=0.01$ (c) $A=1, a=0.08, b=3600, D=0.01$ (d) $A=1, a=0.08, b=3600$, $D=1$ (e) $A=1, a=0.08 b=3600, D=0.00001$ (f) $A=1, a=1, b=1, D=0.01$.

put signal and system output. This will help the weak signal recovery from the noisy system output. Our future work will be directed at extending the initial results of the applications of this scheme in signal processing. 


\section{REFERENCES}

[1] R. Benzi, A. Sutera, And A. Vulpiani, The mechanism of stochastic resonance, J. Phys. A, 14:11(1981), pp. L453-L457.

[2] F. Chapeau-Blondeau, Stochastic resonance and the benefit of noise in nonlinear systems, Noise, Oscillators and Algebraic Randomness - From Noise in Communication Systems to Number Theory, M. Planat, ed., Lecture Notes in Physics, Springer (Berlin), pp. 137-155, 2000.

[3] F. Chapeau-Blondeau and X. Godivier, Theory of stochastic resonance in signal transmission by static nonlinear systems, Phys. Rev. E, 55:2(1997), pp. 1478-1495.

[4] L. Gammaitoni, P. Hänggi, P. Jung, and F. Marchesoni, Stochastic resonance, Reviews of Modern Physics, 70:1(1998), pp. 223-287.

[5] J. J. Collins, C. C. Chow, And T. T. Imhoff, Aperiodic stochastic resonance in excitable systems, Physical Review E, 52:4(1995), pp. 3321-3324.

[6] N. G. Stocks, Suprathreshold stochastic resonance in multilevel threshold systems, Physical Review Letters, 84:11(2000), pp. 2310-2313.

[7] D. Nozaki, D. J. Mar, P. Grigg, and J. J. Collins, Effects of colored noise on stochastic resonance in sensory neurons, Physical Review Letters, 82:11(1999), pp. 2402-2405.

[8] B. Kosko, and S. Mitaim, Robust stochastic resonance: Signal detection and adaptation in impulsive noise, Physical Review E, 64(2001), 051110.

[9] J. J. Collins, C. C. Chow, A. C. Capela, and T. T. Imhoff, Aperiodic stochastic resonance, Physical Review E, 54:5(1996), pp. 5575-5584.

[10] G. Deco And B. Schrmann, Stochastic resonance in the mutual information between input and output spike trains of noisy central neurons, Phys. D, 117(1998), pp. 276-282.

[11] J. J. Collins, T. T. Imhoff, And P. GRigG, Noise-enhanced tactile sensation, Nature, 383:6603(1996), pp. 770 .

[12] N. G. Stocks And D. Allingham, The application of suprathreshold stochastic resonance to cochlear implant coding, Fluctuation and Noise Letters, 2(2002), pp. 169-181.

[13] A. A. Saha and G. V. Anand, Design of detectors based on stochastic resonance, Signal Processing, 83:6(2003), pp. 1193-1212.

[14] X. Godivier and F. Chapeau-Blondeau, Noise-assisted signal transmission in a nonlinear electronic comparator: experiment and theory, Signal Processing, 56(1997), pp. 293-303.

[15] N. G. Stocks, Information transmission in parallel threshold arrays: Suprathreshold Stochastic Resonance, Physical Review E, 63:4(2001), 041114.

[16] F. Chapeau-Blondeau and D. Rousseau, Noise-enhanced performance for an optimal Bayesian estimator, IEEE Transactions on Signal Processing, 52:5(2004), pp. 1327-1334.

[17] B. Xu, F. DuAn, R. BAO, AND J. LI, Stochastic resonance with tuning system parameters: the application of bistable systems in signal processing, Chaos, Solitons and Fractals, 13:4(2002), pp. 633-644, 2002.

[18] B. Xu, F. Duan and F. Chapeau-Blondeau, Comparison of aperiodic stochastic resonance in a bistable system realized by adding noise and by tuning system parameters, Physical Review E, 69(2004), 061110.

[19] T. Wellens, V. Shatokhin, And A. Buchleitner, Stochastic resonance, Reports on Progress in Physics, 67(2004), pp. 45-105.

[20] P. Jung, U. Behn, E. Pantazelou and F. Moss, Collective response in globally coupled bistable systems, Phys. Rev. A, 46:4(1992), pp. 1709-1712.

[21] S. Zozor And P. O. Amblard, Stochastic resonance in discrete time nonlinear AR (1) models, IEEE Transactions on Signal Processing, 47:1(1999), pp. 108-122.

[22] S. Zozor and P. -O. Amblard, On the use of stochastic resonance in sine detection, Signal Processing, 82:3(2002), pp. 353-367. 
18 XINGXING WU, ZHONG-PING JIANG, DANIEL W. REPPERGER, AND YI GUO

[23] B. Xu, J. Li And J. Zheng, How to tune the system parameters to realize stochastic resonance, J. Phys. A: Math. Gen., 36:48(2003), pp. 11969-11980.

[24] P. Jung, Stochastic resonance and optimal design of threshold detectors, Physics Letters A, 207:1(1995), pp. 93-104.

[25] J. Nocedal and S. J. Wright, Numerical Optimization, Springer-Verlag New York, Inc., 1999.

[26] X. Wu, Z. P. JiAng, AND D. W. Repperger, Enhancement of stochastic resonance by tuning system parameters and adding noise simultaneously, Proceedings of the 2006 American Control Conference, pp. 3118-3123, Minneapolis, Minnesota, USA, June 14-16, 2006.

[27] R. L. BADZEy AND R. MOHANTy, Coherent signal amplification in bistable nanomechanical oscillators by stochastic resonance, Nature 437(2005), pp. 995-998.

[28] J. LI AND B. XU, Binary information processing via parameter-induced stochastic reosnance in the presence of multiplicative and additive colored noise with colored cross-correlation, International Journal of Bifurcation Chaos in Applied Sciences and Engineering, 16:2(2006), pp. $427-436$. 